\title{
Anatomical basis of cross sectional echocardiography
}

\author{
NORMAN H SILVERMAN, „ STEWART HUNTER, ROBERT H ANDERSON, $†$ SIEW YEN \\ HO,† GEORGE R SUTHERLAND, $¥$ MICHAEL J DAVIES $†$
}

From the Department of Paediatrics, Cardiothoracic Institute, Brompton Hospital, London; the Department of Cardiology, Freeman Hospital, Newcastle upon Tyne; and the Department of Pathology, St George's Hospital, London

SUMMARY Ten hearts were cut in planes to simulate echocardiographic cuts which may be taken to visualise the anatomy of the cardiac chambers and great arteries. The various myocardial wall segments were named from these sections by referring to their position in the reconstituted heart. The sections were studied in their correct anatomical position. In this way they provide a reference for appropriate orientation of echocardiographic images.

Cross sectional echocardiography is now a well recognised method of cardiac investigation. Indeed, in certain circumstances it has replaced cardiac catheterisation as the primary means of investigation. ${ }^{1-3}$ Recent developments in instrumentation have made it possible to generate echocardiographic images directly comparable with the anatomy of the heart in its natural position within the body and hence as seen by angiocardiography. Complete understanding of these images requires a knowledge of normal tomographic cardiac anatomy. There have been few attempts to section formalin fixed hearts in a fashion directly comparable with echocardiographic images. ${ }^{45}$ Those studies which have been done have tended to display the sections produced in an orientation dictated by the echocardiographic equipment rather than by morphological reality and this has led to comparisons of echocardiograms with sections of hearts orientated in anatomically impossible positions. ${ }^{5}$ In comparison with these correlations using anatomical sections, however, other attempts to correlate anatomy with echocardiography have been inaccurate. This is because they seem to be based exclusively on reconstruction of presumed anatomy from observed echocardiograms rather than by direct correlation with material taken at necropsy. ${ }^{67}$ Consequently there is a need for representation of cardiac anatomy in an orientation which will readily and immediately

\footnotetext{
*Norman H Silverman was visiting professor from the Department of Pediatrics (Cardiology) and the Cardiovascular Research Institute, University of California, San Francisco, USA.

tR H Anderson, Siew Yen Ho, and Michad J Davies are supported by the British Heart Foundation.

$\ddagger$ George R Sutherland is now consultant paediatric cardiologist at the Wessex Regional Cardiothoracic Centre, Southampton.

Accepted for publication 5 July 1983
}

be of value not only to the cross sectional echocardiographer but to all who study the image.

\section{Materials}

Ten hearts from people who died without any known structural heart disease were fixed by perfusion with buffered $10 \%$ formalin to preserve their three dimensional integrity. Each heart was then cut with a sharp brain knife to simulate one of the series of planes used during cross sectional echocardiography (see below). In each series of cuts the heart was placed in its anatomical position. After each cut the heart was photographed. In several instances the sections of heart were reconstituted using cyanoacrylate glue (Superglue) and the heart was then resectioned in a different series of planes.

\section{ARRANGEMENT OF THE CARDIAC CHAMBERS}

To understand the sectional anatomy it was first necessary to appreciate the disposition of the cardiac chambers relative to the position of the heart in situ. ${ }^{8}$ The long axis of the heart deviates considerably from the long axis of the body, the cardiac axis being from the left hypochondrium (apex) towards the right shoulder (base) (Fig. 1). Within the frontal cardiac silhouette the right sided cardiac chambers (right atrium and right ventricle) are positioned considerably anterior to their left sided counterparts, while the ventricles are placed inferior and leftward relative to their corresponding atria. The pulmonary valve is positioned leftward and superior to the aortic valve. Consequently, the right ventricle occupies the greater part of the anterior surface of the cardiac silhouette, extending from the inferiorly placed tricuspid valve to 


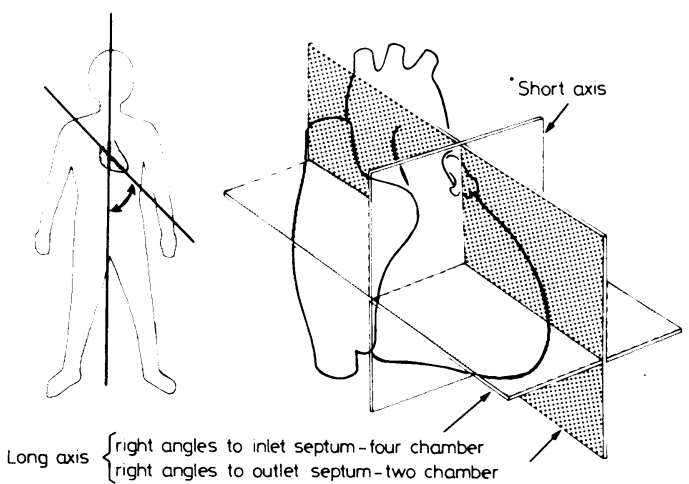

Fig. 1 The long axis of the heart is inclined at about $45^{\circ}$ to the long axis of the body in terms of three orthogonal planes.

the superiorly located pulmonary valve (Figs. 2a and b). Because of this "wrap around" relation of the right ventricle to the left ventricle, the different components of the ventricular septum occupy various planes. We find it easier to describe these interrelationships by considering both the ventricles themselves and the muscular interventricular septum as having three components, rather than the traditional "sinus" and "conus." These components are the inlet component, extending from the atrioventricular annulus to the distal attachment of the papillary muscles, the apical trabecular component, and the outlet component which supports the arterial valve. The inlet septum (between the inlet portions) is then placed at an angle of almost $90^{\circ}$ to the outlet part of the septum. The apical trabecular septum curves round between these two extremes in addition to extending out between the ventricular apices (Fig. 3).

The ventricular septum is thus well described in terms of its three muscular components, these radiating out from the small fibrous or membranous com-
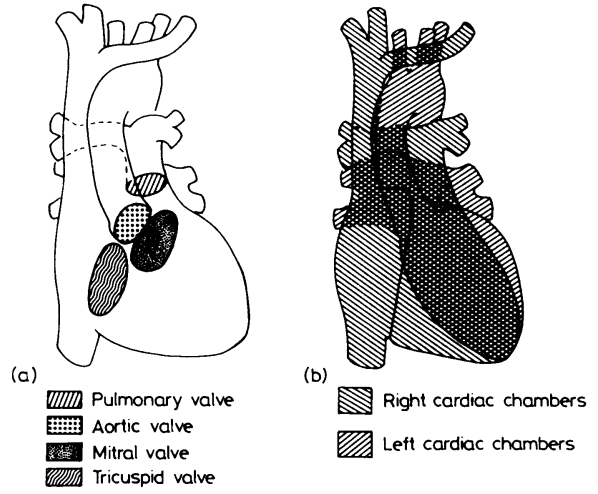

Fig. 2 Positions of the four cardiac valves as seen in the frontal cardiac silhouette $(a)$ are related to the fact that the right sided chambers are anterior to their left sided counterparts $(b)$.

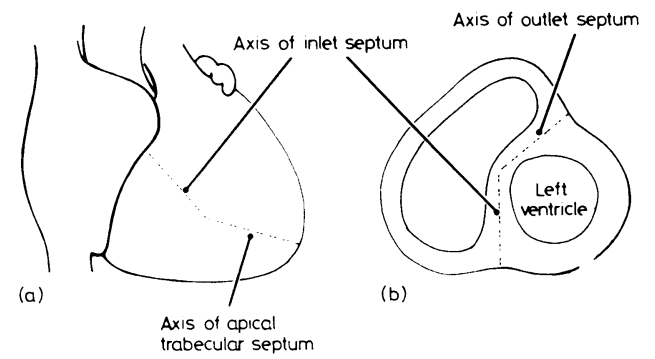

Fig. 3 Different angles of the components of the ventricular septum as seen from the front (a) (axis of outlet septon parallel to this projection) and in the short axis (b) (trabecular septon shows similar curve in apical sections).

ponent which is part of the central fibrous body (the junction of aortic, mitral, and tricuspid valves). The ventricular free walls are also well described in terms of their inlet, apical trabecular, and outlet components. Supplementary description is then provided by recognising the surfaces of the ventricular mass. When viewed from its apex looking towards the base the mass is roughly triangular (Fig. .4a). One surface rests on the diaphragm and is conveniently designated the diaphragmatic surface. There is a abrupt margin between this surface and the anterior sternocostal surface. This acute margin is the inferior side of the mass in anterior projection (Fig. 4b). The other two margins are much more gradual, being between the pleural surface of the heart and the sternocostal and diaphragmatic surfaces respectively. The upper of these margins forms the right hand side of the anterior silhouette and is the obtuse or left lateral margin of the left ventricle (Fig. 4b). On the posterior surface of the ventricular mass, the point at which the plane of the inlet ventricular septum intersects with the atrioventricular junction is termed the crux. The Table summarises the terms used to describe the heart.

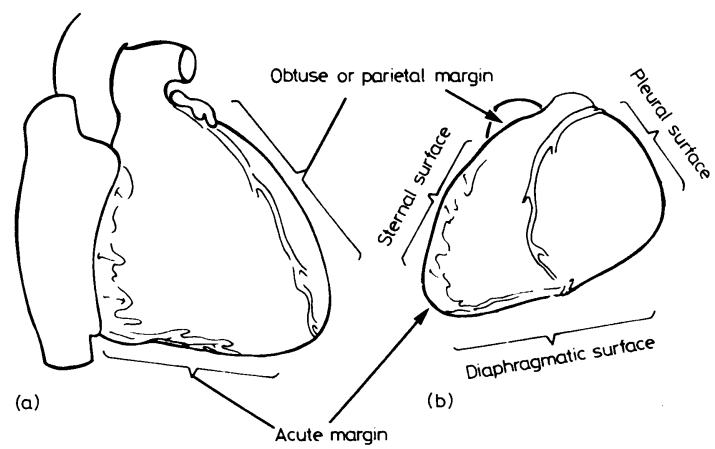

Fig. 4 Surfaces and borders of the cardiac silhouette seen in frontal projection (a) and in the short axis (b). 
Table Approach to description of the heart

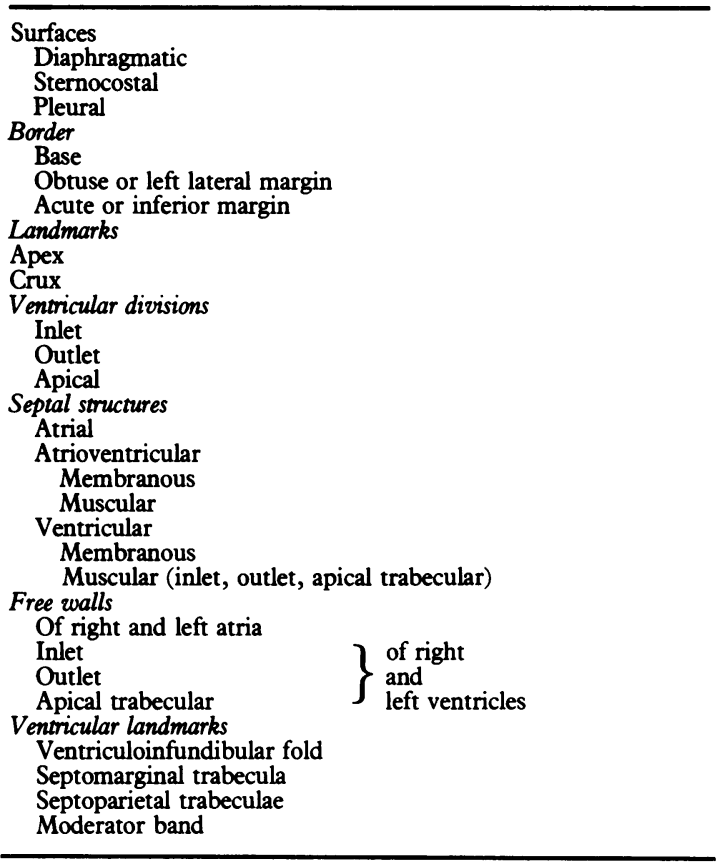

\section{ECHOCARDIOGRAPHIC APPROACHES TO THE}

HEART

Full description of any three dimensional structure requires recognition of its three orthogonal planes. In the body these are the sagittal and coronal long axis planes and the horizontal or short axis plane (Fig. 5). As indicated above, the long axis of the heart is aligned at an angle relative to the long axes of the body

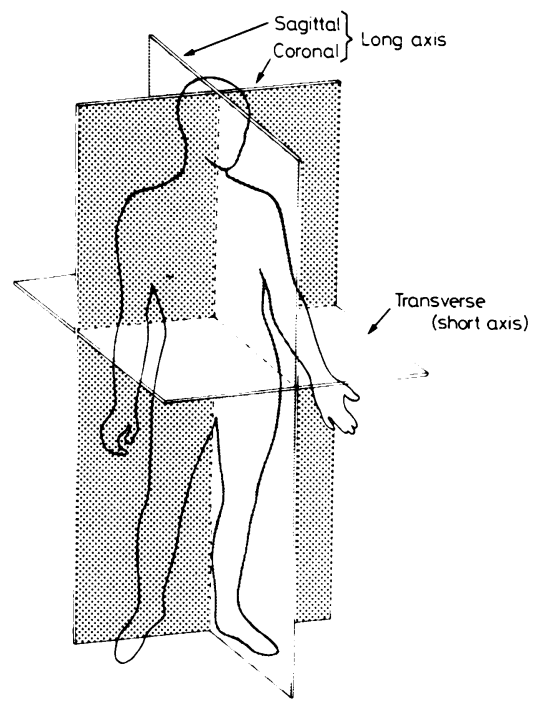

Fig. 5 The three orthogonal planes of the body.
(Fig. 1). None the less, the heart itself can also be described in terms of three orthogonal planes (Fig. 1). The two long axis index planes are at right angles to the inlet and outlet parts of the ventricular septum. The plane through the inlet septum shows both atrial and both ventricular chambers and is called the four chamber plane. The plane parallel to the outlet septum has traditionally been called simply the long axis plane, but this convention obscures the fact that the four chamber plane is also in the cardiac long axis. As we will see, the series of planes approximately at right angles to the outlet septum (parallel to the inlet septum) show for the most part either the two right sided or the two left sided chambers. For this reason we propose to refer to these planes as two chamber planes, following the precedent of Edwards et al ${ }^{9}$ The two chambers are often supplemented by a great artery or its outflow tract. The third series of planes are the short axis planes, which are at right angles to both four chamber and two chamber series.

When demonstrating the cross sectional anatomy of these planes, the echocardiographer is constrained by the fact that the heart is largely overlain by the lungs and the entire thoracic organs are encased by the rib cage. Access is therefore limited to the echocardiographic windows. These are the parasternal, apical, subcostal, and suprasternal portals (Fig. 6). From each of these windows the investigator can obtain two of the three orthogonal planes, together with a series of intermediate planes between these two and towards the third plane. The third index plane can never be visualised because the transducer is itself in that plane.

From the parasternal interspaces, cuts in the two chamber and short axis planes of the heart itself can be obtained (Fig. 7a). From the apical position, the cuts can be taken in both long axis planes of the heart, yielding the four chamber and two chamber series respectively (Fig. 7b). From the subcostal or suprasternal position, it is not normally possible to manipulate the transducer along the cardiac orthogonal axes. Instead the plane of the sound beam is moved along the axes of the body, producing parasagittal and paracoronal series (Fig. 7c). As we will see, these can approximate to the long and short axis cuts of the heart, but there are appreciable differences in the information provided.

\section{ORIENTATION OF THE CARDIAC SECTIONS}

Up to date echocardiographic machines now have the facility to display the image provided in the orientation selected by the operator. We believe that there is every reason now for doing this in the same fashion as for most other cardiac investigations. Thus a patient is almost always examined from the front with the right side of the patient to the observer's left hand. Frontal 


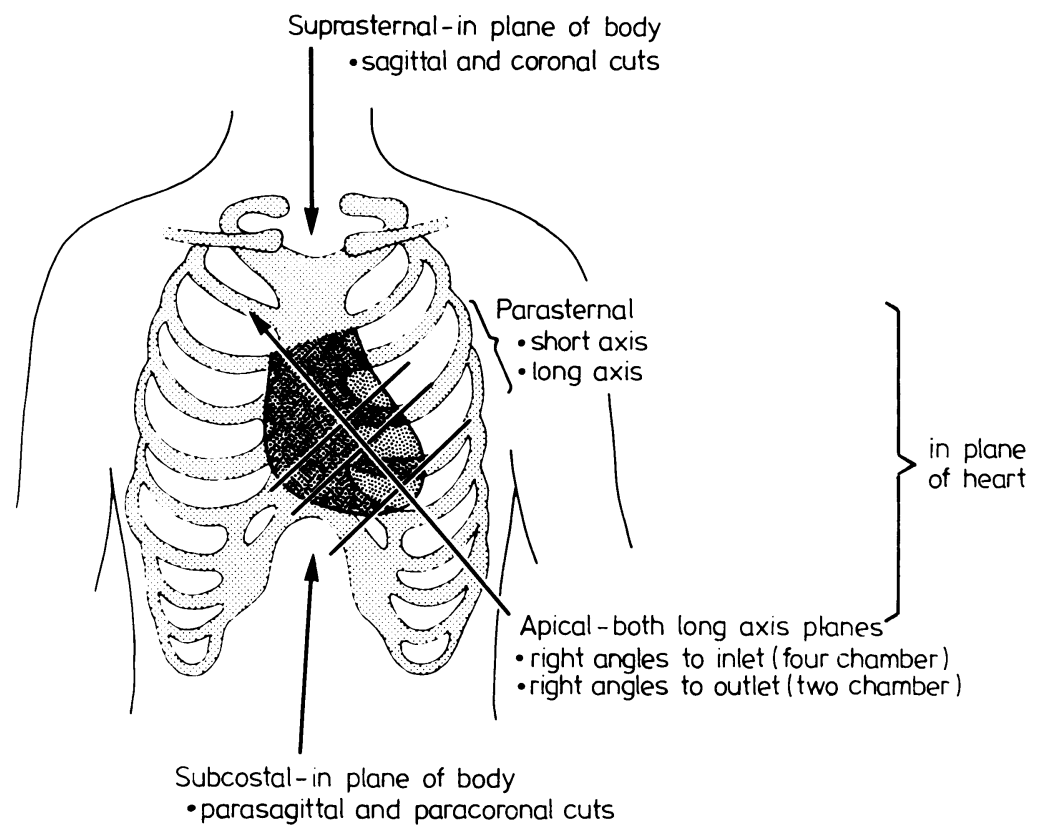

Fig. 6 The echocardiographic windows determine whether the reference axes are those of the heart or those of the body.

chest radiograms are almost always presented in similar fashion, as are angiocardiograms. Anatomical sections taken in the four chamber plane are then readily understood when orientated with the atria upwards and to the right and the ventricular apex downwards and to the left. When viewing the two chamber series it is less important whether the anterior surface of the heart is to the left or the right hand. None the less, it is comparable with the usual demonstration of left lateral cardiac radiographs to place the anterior surface towards the left and the posterior surface towards the right. This means placing the apex to the left hand. It still aids interpretation for those not experienced in echocardiography, however, if, when posible, sections are positioned with the atria above the ventricles. When considering the orientation of short axis sections, these can then be made comparable with the frontal projection by viewing the section from the cardiac apex looking towards the base. The right side of the heart is then seen to the observer's left hand with the anterior sternocostal surface uppermost.

\section{Results}

SECTIONS SIMULATING VIEWS FROM THE APICAL WINDOW

Both the apical index planes are in the long axis of the heart. Two of the hearts were cut in the two chamber plane and four hearts were cut in the four chamber plane. The index plane at right angles to the outlet septum and parallel to the inlet septum is the two chamber section, as are the rightward and leftward series of parallel sections (Fig. 8).

The index section (Fig. $9 \mathrm{~b}$ ) passes through the ventricular apex and up through the aorta. It is parallel to the inlet septum and at right angles to the outlet septum. It shows mostly the left ventricle, with the small outlet part of the right ventricle seen anteriorly. The transected wall of the left ventricle is the diaphragmatic surface of the inlet and trabecular components. Posteriorly the cut shows the anterior and diaphragmatic surface of the left atrium. The feature of aortic mitral valve fibrous continuity is well illustrated. The parallel two chamber sections to the right and left of the index section show the right sided and left sided cardiac chambers respectively (Fig. 9a and 9c). These cuts are parallel to the greater part of the ventricular septum, which is not visualised in the right sided cut nor in the left sided one when this is taken to the left of the pulmonary trunk. The other long axis apical section is at right angles to the inlet septum and parallel to the outlet septum. The most anterior of these sections usually obtainable echocardiographically shows the origin of the aorta from the left ventricle and the ventriculoinfundibular fold which separates the right ventricular outflow from the tricuspid valve (Fig. 10a). A deeper cut then shows the posterior aspect of the left ventricular outflow tract wedged between the mitral valve and the septum (Fig. 10b). This and slightly deeper sections show the site of the small membranous component of the ventricular septum and illustrate the acute and left lateral surfaces of 
(a)

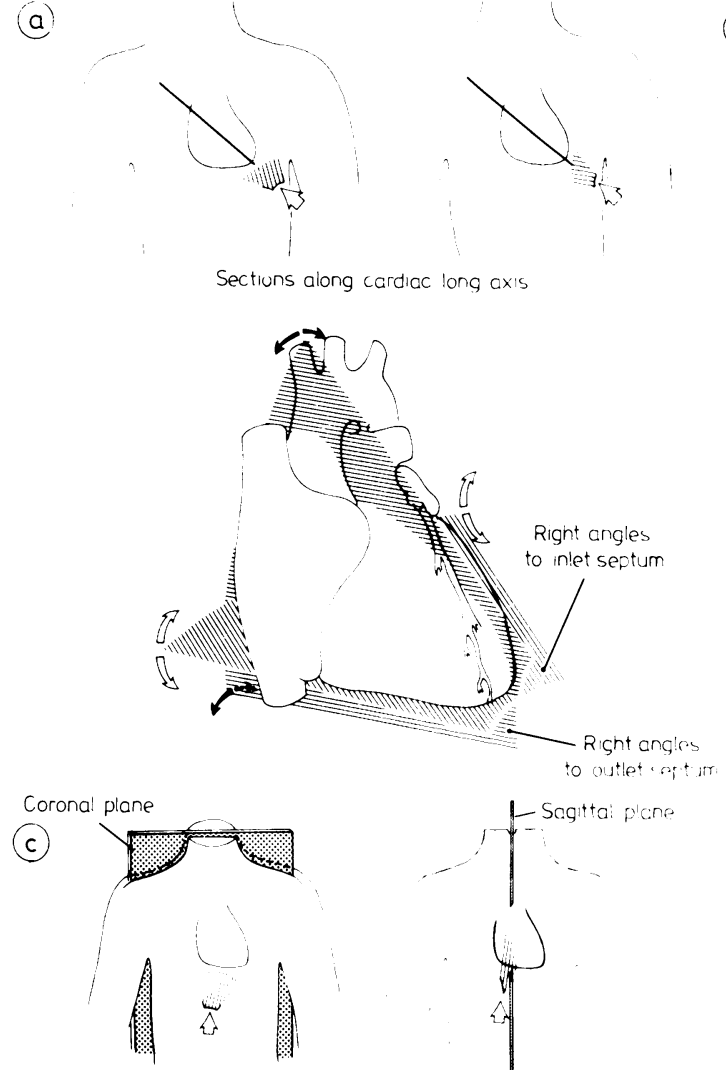

Paracoronal four chamber cuts
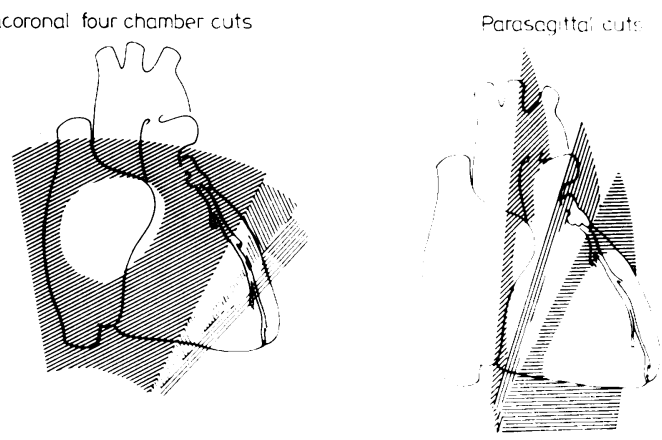

the ventricular mass. A deeper cut behind the aortic root then shows the four cardiac chambers, the different levels of attachment of the atrioventricular valves producing the atrioventricular muscular septum (Fig. 10c). The presence of septal attachments of the tricuspid valve and absence of mitral septal attachments are shown, as is the differing morphology of the right sided and left sided atrioventricular junctions. It is the inlet and trabecular components of the ventricular septum that are visualised, together with the oval fossa of the atrial septum and its surrounds. The most

Fig. 7 Scanning from the cardiac apex (a) produces a series of cuts in long axes planes at right angles to the inlet (four chamber) or to the outlet (two chamber) components of the ventricular septum. The arrows indicate the series of planes radiating from the index. Scanning from the parasternal window (b) permits the heart to be cut in its own short axis or long axis plane. Scanning from the subcostal plane (c) produces a series of cuts in the paracoronal or parasagittal planes of the body.

posterior of the four chamber cuts is through the crux of the heart (Fig. 10). This shows the intersection of the inlet ventricular septum with the atrioventricular junction and illustrates the coronary sinus draining through the left atrioventricular groove into the right atrium.

\section{SECTIONS SIMULATING VIEWS FROM THE}

PARASTERNAL WINDOW

Three of the 10 hearts were sectioned to simulate the parasternal long axis plane. This produces a series of 


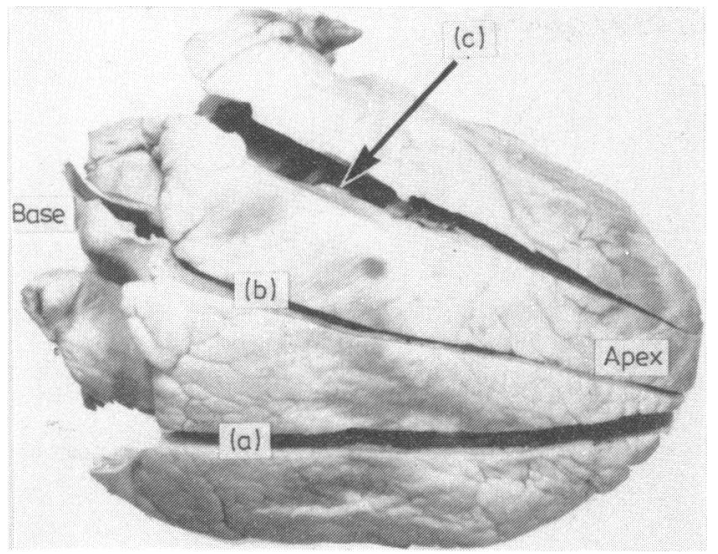

Fig. 8 The three long axes cuts at right angles to the outlet septum which produce the two chamber series of echocardiographic planes.

two chamber cuts which, in terms of their anatomy, are directly comparable with the apical two chamber sections. From the standpoint of echocardiography, however, the sections are orientated with the apex to the observer's left hand.

The second series of parasternal cuts produces short axis sections. These show well the sweep of the ventricular septum and illustrate the angulation between its inlet and outlet components. The apical sections illustrate well the coarsely trabeculated right ventricle and the circular apical part of the left ventri- cle (Fig. 11a). The cut at mid-ventricular level transects the mitral valve papillary muscles (Fig. 11b). Parallel cuts taken through a higher interspace show the wedged position of the aortic valve and subaortic outflow tract together with the extensive ventriculoinfundibular fold interposed between the atrioventricular and arterial valves of the right ventricle (Figs. 1lc and 11d). The different morphology of the atrioventricular valves is also well illustrated, particularly the septal attachments of the tricuspid valve and the discrete plane between the left ventricular septal surface and the mitral valve.

\section{SECTIONS SIMULATING VIEWS FROM THE SUBCOSTAL WINDOW}

From the subcostal window the sound beam when directed through the heart traverses it in the paracoronal and parasagittal planes of the body rather than in the axes of the heart itself (Fig. 7c). Two hearts were sectioned in each of these planes. The two series of cuts have much in common with the four chamber and short axis series, although the long axis cuts also provide two chamber planes depending on the extent to which the transducer can be advanced beneath the rib cage. Generally, especially in younger people, in whom these planes are more readily achieved because of lax abdominal muscular tone, the fulcrum of the long axis paracoronal planes will be on or posterior to the acute margin of the right ventricle. Then the anterior cut will enter through the acute marginal surface and will provide a two chamber section of the
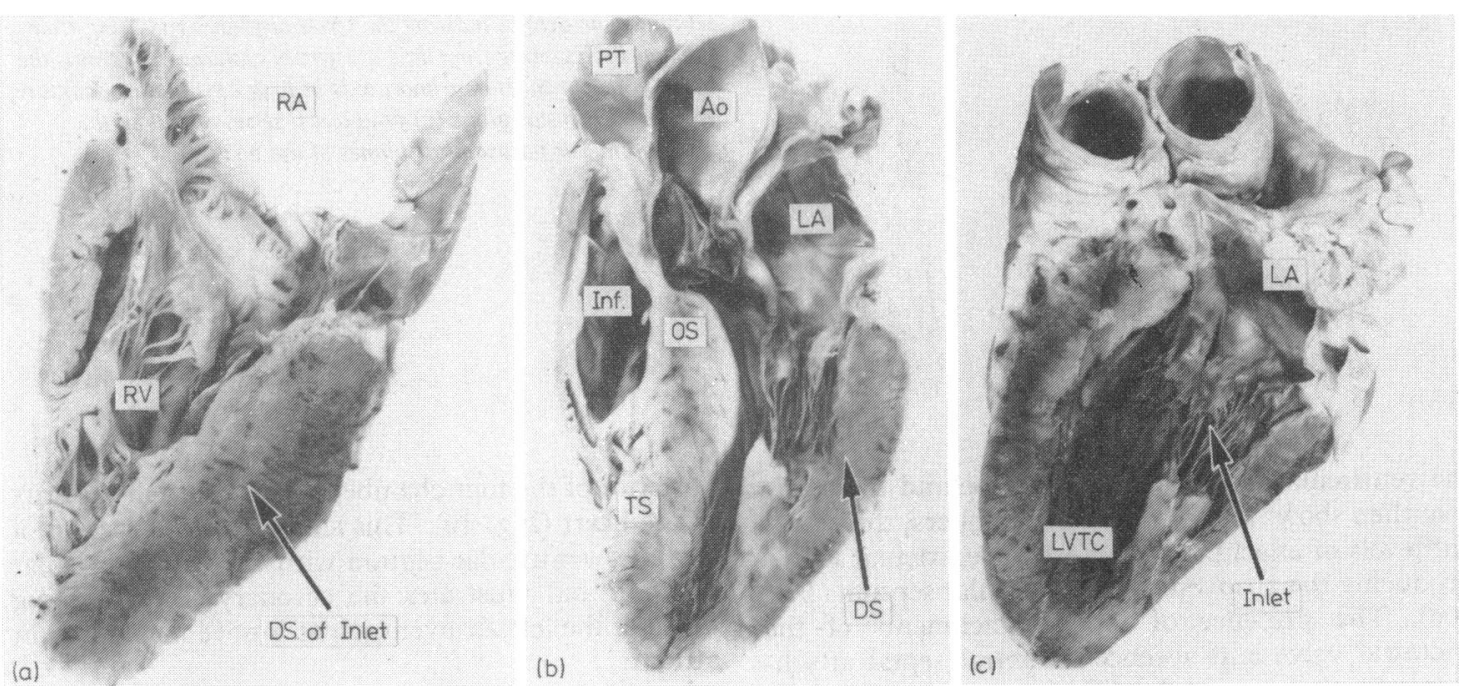

Fig. 9 The index two chamber long axis section obtainable from either the apical or parasternal windows (a). The radiating right sided two chamber cut showing the right atrium, tricuspid valve, and right ventricle (b). The radiating left two chamber cut showing the left atrium, left ventricle, and anteriorly situated right ventricular oufflow tract (c). RA, right atrium; $R V$, right ventricle; $D S$, diaphragmatic surface; PT, pulmonary brink; Ao, aorta; LA, left atrium; Inf, infundibulum; OS, outlet septum; TS, trabecular septum; $L V$, left ventricle; $T C$, trabecular component. 

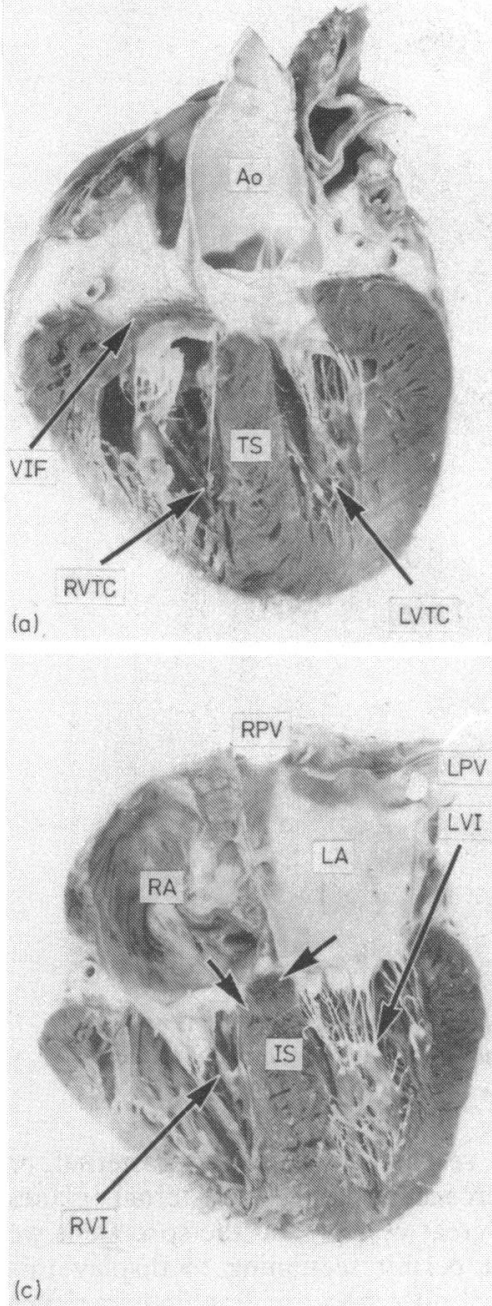

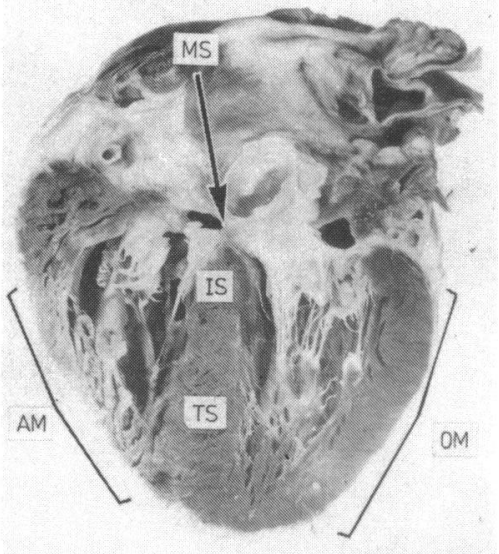

(b)

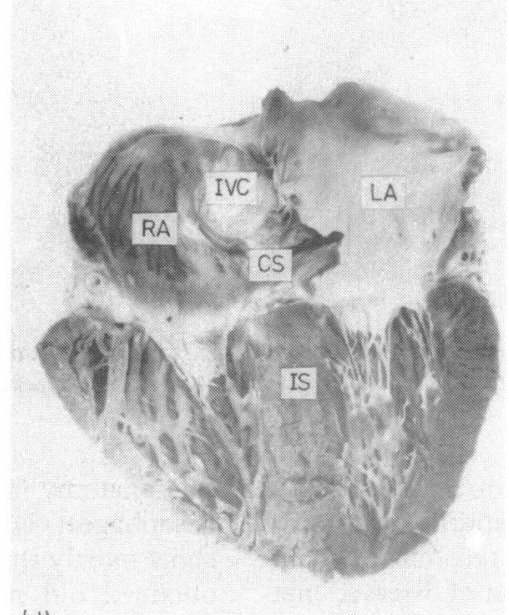

Fig. 10 Four of a series of apical four chamber cuts. VIF, ventriculoinfundibular fold, IS, inlet septum; $M S$, membranous septum; $A M$, acute margin; $O M$, obtuse margin; I, inlet; $P V$, pulmonary vein, CS, coronary sinus; IVC, inferior vena cava. right ventricle extending up to the pulmonary trunk (Fig. 12a). A deeper section will cut through the ventriculoinfundibular fold and extend into the subaortic ouflow tract, visualising the inlet and trabecular parts of the septum and the obtuse margin of the left ventricle (Fig. 12b). Sectioning still more deeply will approximate to the four chamber apical section (Fig. 12c). The deepest section of all is particularly important since this can cut behind the inlet part of the ventricular septum while still passing through the atrial septum. This produces a three chamber section which superficially suggests absence of the right atrioventricular connection (Fig. 12d). The full series of paracoronal sections (Fig. 12a-d) are needed to elucidate fully the anatomy.

The parasagittal sections approximate to the short axis cardiac sections (Fig. 13a). The angle of inci- dence is such that the outlet part of the septum is shown to greater advantage (Fig. 13b and 13d). Angling the cuts from this section then shows the important relation between the subaortic and subpulmonary outflow tracts and the wedge position of the aortic valve between the tricuspid and mitral valves (Fig. 13c). The morphology of the central fibrous body is well illustrated in this series of cuts, as is the arrangement and integrity of the atrial septum (Fig. 14a and 14b).

\section{Discussion}

A knowledge of cardiac anatomy and pathology as seen in cross section is essential for understanding echocardiography, as it is for other cross sectional techniques such as computed tomography and nuclear magnetic resonance. The sectional presentation of 

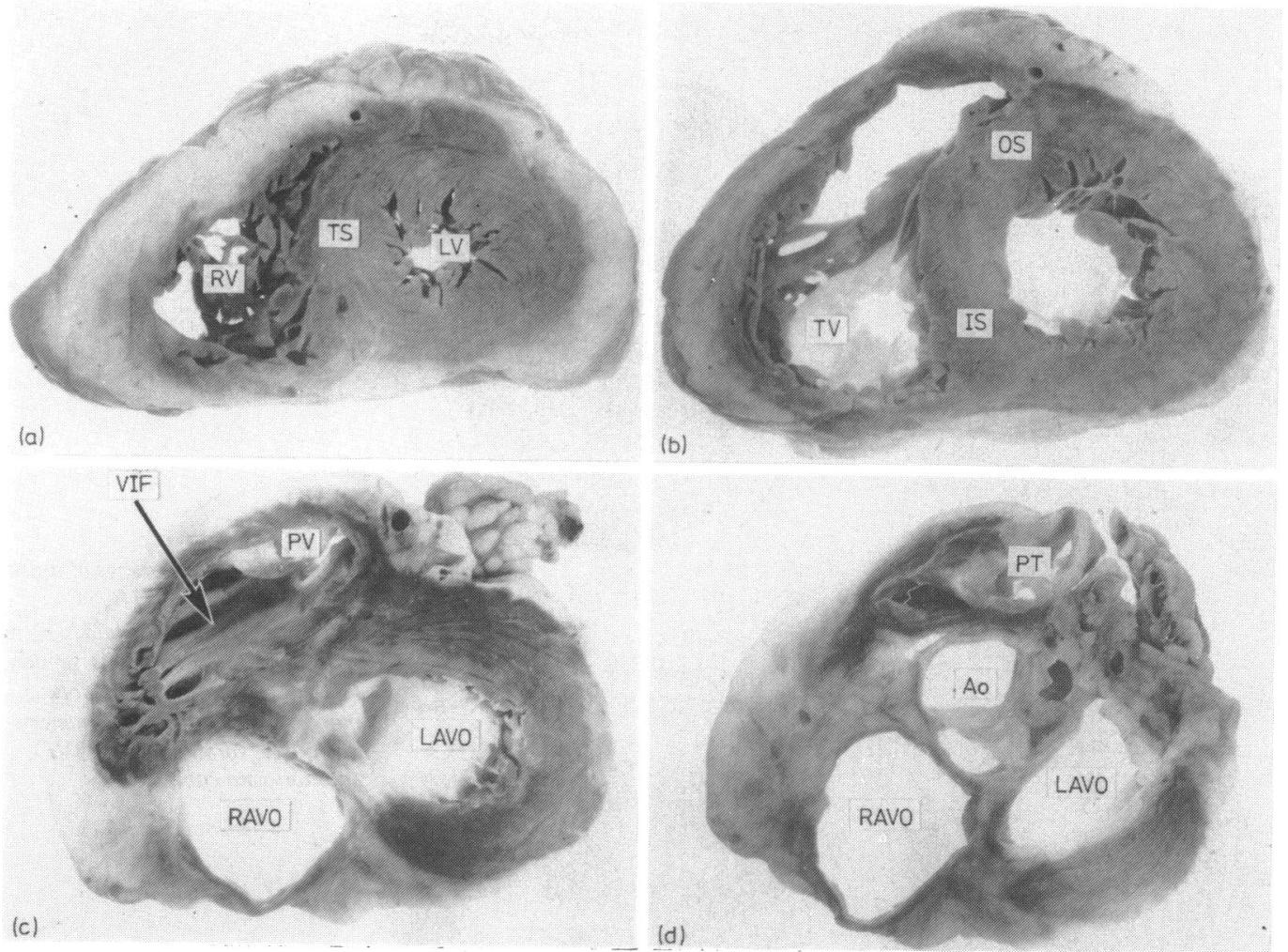

Fig. 11 Four of a series of short axis cuts as would be obtained from parasternal short axis cuts. TV, tricuspid valve; $P V$, pulmonary valve; $L A V O$, lefi atrioventricular orifice; $R A V O$, right atrioventricular orifice. For other abbreviations see Figs. 9 and 10.

cardiac anatomy displayed here is a direct extension of the detailed and accurate second report of the Mayo Clinic group. ${ }^{9}$ Like them, we also recommend preparation not only of normal hearts but of diseased material in a way directly comparable with the echocardiographic images. The use of cyanoacrylate (Superglue) means that this need no longer obviate examination in "standard" pathological fashion. Clean cuts in the heart can be readily and completely repaired with the glue, thus permitting sectioning after the preliminary diagnostic examination. Furthermore, skilful use of the glue enables the hearts to be reconstituted after sectioning and resectioned in different planes.

We have described our sections in terms of the three orthogonal planes of the heart, deviating from this only when the echocardiographic access is such that the sound beam must be scanned in the orthogonal planes of the body rather than the heart itself. We have displayed our sections so that the cardiac anatomy is presented in the setting of the heart within the body. In this respect we have illustrated only the views of the heart obtained from parasternal, apical, and subcostal windows. We have not shown the anatomy as seen from either suprasternal or oesophageal approaches. The suprasternal planes show mostly the great vessels, and the specimens we obtained did not permit sectioning to display this anatomy. We have no echocardiographic experience using the oesophageal portal.

Our sections and their orientation are important in the light of technological advances which make it possible for echocardiographic images to be displayed in the way desired by the operator. There are good reasons for using this facility to display cardiac anatomy as dictated by the position of the heart within the chest. Firstly, the images displayed in this way are readily understandable not only to the operators of the echocardiograph but also to their colleagues, both clinical and paraclinical. How many times have uninitiated observers been mystified by an apparently unconventional display of echocardiographic material? This would not be necessary if the images were displayed as we have shown the anatomical sections in this review. This is the expected orientation, and the pictures obtained are immediately compatible with those obtained using more familiar investigative tech- 

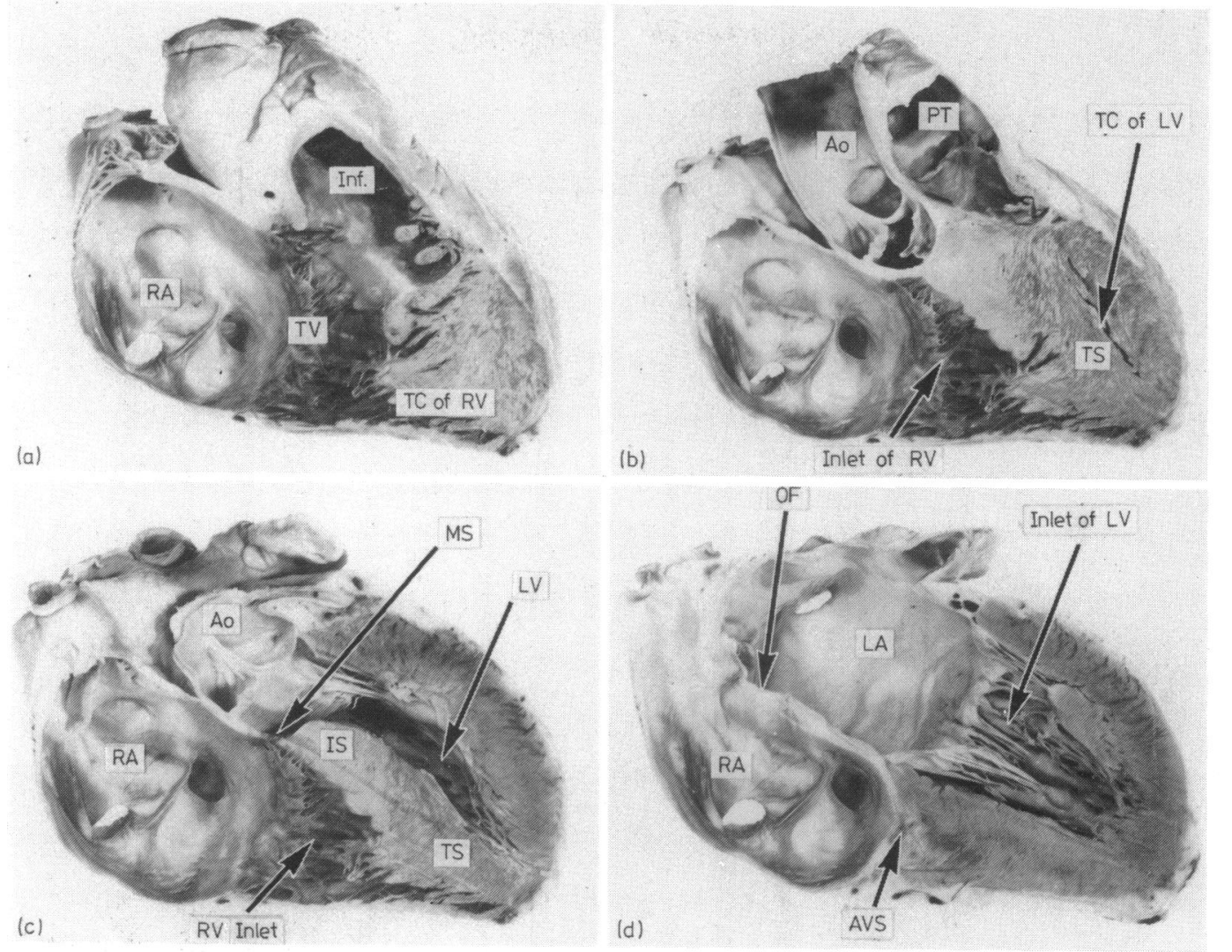

Fig. 12 Four of a series of subcostal cuts taken in the paracoronal plane. OF, oval fossa; AVS, atrioventricular sulcus. For other abbreviations see Figs. 9 and 10.

niques. The second reason relates to the ease with which the operator himself can scan the heart. Previous experience attests to the skill of echocardiographic investigators who have unravelled the intricacies of cardiac anatomy when displayed in most unanatomical positions. How much easier this would be if the image were presented in a way which could immediately be thought of in terms of the heart within the body. Our own echocardiographic experience since starting to use this orientation suggests that more information will become available and will be obtained more quickly than using the unanatomical alternatives. The orientations we have used in this review are recommended as one valid alternative by the American Society of Echocardiography. ${ }^{10}$ The ease with which the anatomical features can be recognised echocardiographically when presented in the suggested orientation, coupled with the facility it provides for direct comparison with other imaging techniques, indicates that it should be the only valid option.
We have used a purely descriptive approach for the account of the sections. This is valid not only for description of the cardiac chambers but also for the ventricular "segments." These are of considerable importance to investigators interested in abnormal movement of the ventricular walls and the relation of this dyskinesia to myocardial ischaemia and disease. Workers in these fields have constructed formidable arbitrary conventions, almost all indicated in numerical fashion, in which the ventricles have been divided into between five ${ }^{11^{-13}}$ and $30^{14}$ segments. Such procrustean division is neither necessary nor helpful. The nature of the divisions are related neither to anatomical landmarks nor to the pattern of ventricular arterial supply. The information concerning abnormal motion of the ventricular walls can be conveyed by describing the septal, diaphragmatic, and free wall surfaces of the inlet, apical, and trabecular components respectively. This can then be readily interpreted in terms of the course of the coronary arteries. If quantitation is needed this can be done directly for any selected 

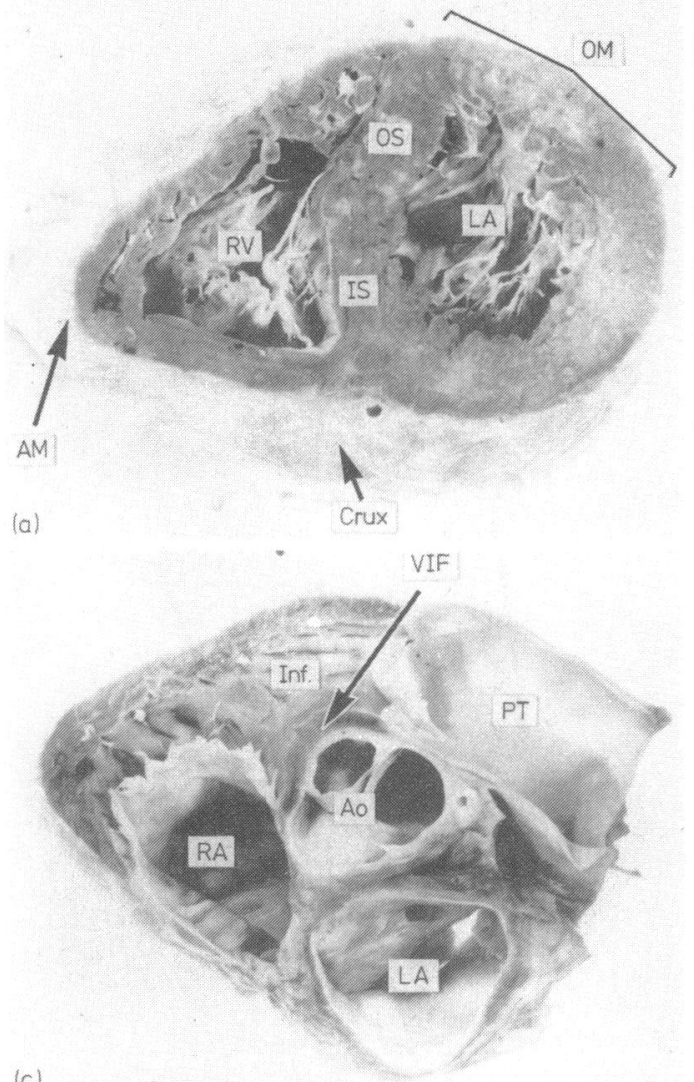

Fig. 13 Four of a series of parasagittal short axis subcostal cuts. Cut is at mid-ventricular level. Note the similarity to Fig. 11b. Fig. $13 \mathrm{~b}$ shows the outlet ventricular septum and shows the wedged position of the aortic root. Compare this with Fig. 11d. Fig. 13d is rotated slightly more to the right to show the plateau of the pulmonary and tricuspid valves to the aortic root. SVC, superior vena cava. For other abbreviations see Figs. 9 and 10.

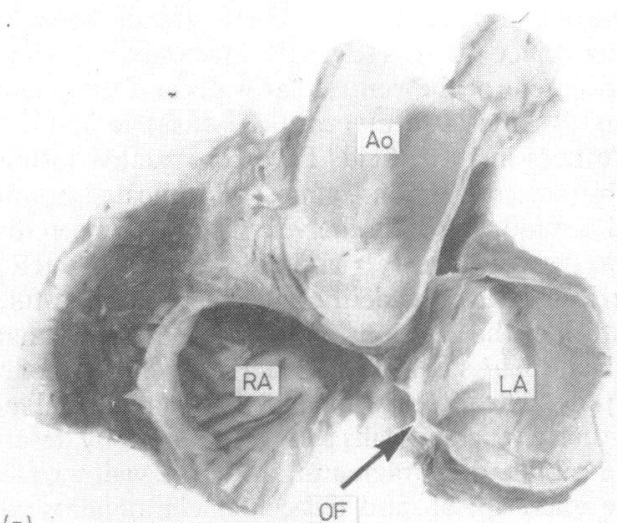

(a)

of

Fig. 14 Subcostal and parasagittal cuts showing the atrial septum. Fig. 14a shows that part related to the aortic root and (b) shows the surrounds of the oval fossa. TC, terminal crest. For other abbreviations see Figs. 9 and 10. 
echocardiographic section rather than interposing the arbitrary "grid system" suggested by Edwards et al. 9 and modified by the American Society of Echocardiography. ${ }^{10}$ Even if a system of computer coordinates for myocardial segments is used these can be described better in anatomical terms than by numerical symbols.

In our study we prepared sections in the three orthogonal axes only. We appreciate that the planes chosen provide a reference in merely one particular scan plane. The echocardiographer has the facility (and uses it constantly) to make many "off axis" cuts of the heart with minor changes of the transducer position. We have not attempted to display the specimens in these oblique planes, since they are almost infinite. None the less, in choosing the triaxial system of coordinates here recommended, certain caveats should be noted. If the heart is examined using cuts which entail sweeping along the axes of the body, then differences in position and orientation of the heart will considerably alter the anatomy displayed. For example, the views obtained when the heart is right sided (dextrocardia) will bear little resemblance to those illustrated here, even if the heart is structurally normal. Similarly, when the heart is malpositioned, quite different transducer positions will be needed to display the stimulated echocardiographic sections we have illustrated. In short, the sections we have simulated provide only the basis for understanding. For precision, the echocardiographer should describe his results using a system based on transducer position, the plane obtained, and the modification of this plane by manoeuvres such as cranial angulation or oblique rotation.

\section{References}

1 St John Sutton MG, St John Sutton MB, Oldershaw P, et al. Valve replacement without preoperative cardiac catheterization. $N$ Engl f Med 1981; 305: 1233-8.

2 Stark J, Smallhorn J, Huhta J, et al. Surgery for congenital heart defects diagnosed with real-time echocardiography [Abstract]. Circulation 1982; 66 (suppl II): II-30.

3 Rice MJ, Seward JB, Hagler DJ, et al. The impact of two-dimensional echocardiography on the management of the distressed newborn suspected of having cardiac diseases [Abstract]. Circulation 1982; 66 (suppl 2): 231.

4 Tajik AJ, Seward JB, Hagler DJ, Mair DD, Lie JT. Two-dimensional real-time ultrasonic imaging of the heart and great vessels. Technique, image orientation, structure identification, and validation. Mayo Clin Proc 1978; 53: 271-303.

5 Yuste P, Garcia-Fernandez MA. Atlas de ecocardiografia. Madrid: Ediciones Norma, 1982.

6 Silverman NH, Schiller NB. Apex echocardiography. A two-dimensional technique for evaluating congenital heart disease. Circulation 1978; 57: 503-11.

7 Sahn DJ, Anderson F. Two-dimensional anatomy of the heart. New York: John Wiley, 1982.

8 Anderson RH, Becker AE. Cardiac anatomy-an integrated text and colour atlas. London and Edinburgh: Churchill Livingstone, 1980.

9 Edwards WD, Tajik AJ, Seward JB. Standardized nomenclature and anatomic basis for regional tomographic analysis of the heart. Mayo Clin Proc 1981; 56: 479-97.

10 Henry WL, DeMaria A, Gramiak R, et al. Report of the American Society of Echocardiography committee on nomenclature and standards in two-dimensional echocardiography. Circulation 1980; 62: 212-7.

11 Weyman AE, Peskoe SM, Williams ES, Dillon JC, Feigenbaum $\mathrm{H}$. Detection of left ventricular aneurysms by cross-sectional echocardiography. Circulation 1976; 54: $936-44$.

12 Kisslo JA, Robertson D, Gilbert BW, von Ramm O, Behar VS. A comparison of real-time, two-dimensional echocardiography and cineangiography in detecting left ventricular asynergy. Circulation 1977; 55: 134-41.

13 Horowitz RS, Morganroth J, Parrotto C, Chen CC, Soffer J, Pauletto FJ. Immediate diagnosis of acute myocardial infarction by two-dimensional echocardiography. Circulation 1982; 65: 323-9.

14 Visser CA, Kan G, Lie KI, Becker AE, Durrer D. Apex two dimensional echocardiography. Alternative approach to quantification of acute myocardial infarction. Br Heart F 1982; 47: 461-7.

Requests for reprints to Professor Robert H Anderson, Department of Paediatrics, Cardiothoracic Institute, Brompton Hospital, Fulham Road, London SW3 6HP. 